\title{
Conducting Contextual Inquiry of Twitter for Work Engagement: An Indian Perspective
}

\author{
Nikhil Wani \\ Vishwakarma Institute of \\ Technology. \\ Pune, India
}

\author{
Ganesh Bhutkar \\ Indian Institute of Technology \\ (IIT), Bombay \\ Mumbai, India
}

\author{
Shreeya Ekal \\ Vishwakarma Institute of \\ Technology \\ Pune, India
}

\begin{abstract}
This research paper presents a comprehensive Contextual Inquiry (CI) of Indian Twitter users and then proposes related work models with interesting insights into aspects that contribute to their work engagement. The study involved observing the users interact with Twitter in their workplaces, which has helped in understanding their behavioral preferences for an engaging work environment. Furthermore, a series of structured contextual interviews and onsite visits were carried out with a convenient sampling of 18 users from diverse environments. A random sampling of 2,000 Indian users was programmatically derived and analyzed which reflects the essence of Twitter usage in work environments. The resultant data was documented for advancing to the second-order analysis, which strengthens the premises of the four proposed work models. They aim at developing and communicating a better understanding of Indian Twitter users to facilitate future contextual designs. In addition, the paper analyzes the genuine usability issues that prevent a smooth design for work engagement. It also identifies the opportunities for understanding global and local cultural differences of Twitter users. Looking into the future, we revisit Twitter's design architecture and derive key aspects that extend the dimensions of work engagement leading to facilitation of apt business strategies for Indian Twitter users. In future, this CI study will form the basis for work engaging Twitter bots that adhere to ethnographic strategies during their service to regional users.
\end{abstract}

\section{General Terms}

Human Computer Interaction - Field studies.

\section{Keywords}

Contextual Inquiry; Work Engagement; Ethnographic study Indian Users; Twitter; Work Models; Data Analytics.

\section{INTRODUCTION}

Twitter, which started out as a microblogging service in 2006, once reserved for cheeky and banal mini-posts [30], has now turned into a star studded and potential work engagement platform with 310 million monthly active users [27]. Twitter is used by users to publicly express their identity and personality. At times, it tends to transform into a catalyst for public discussions, by breaking the news and happenings before the traditional media knows about it. The study of Tweeple, the coined term for Twitter using people, has been of vital importance in context of Indians, since research suggests that Indian users spend on average 8.4 hours every day on social media [17]. Twitter's official announcement about their users proclaimed that as of $2017,79 \%$ of Twitter accounts have been created outside the United States [7] and hence, become an important subject for ethnographic observations and quintessential study for better work engagement.

In this paper, Contextual Inquiry (CI) is conducted for better understanding of Twitter as a potential platform for enhancing work engagement by common Indian employees working for IT as well as non-IT companies. It provides a unique way to advance this analysis by gathering detailed and essential field data, which involves actual visits to the workplaces or companies of these employees, who are also active Twitter users. Convenient sample of 18 users used for this paper were employees of IT companies, while a major subset of random sampled 2,000 users belonged to non ITcompanies considering an unbiased culmination of results. Work engagement being a very new and intriguing dimension of workplace psychology serves as the basis of fulfilling and productive work [1]. Visiting and observing the users interact with the product in their workplace helps understand and examine their work preferences. These on field preferences are collectively noted with a very simple, but powerful technique called Affinity Diagramming. These diagrams provide a good way for grouping and understanding information [4] from all the visits made to the users.

Marketers build on quantitative data, while designers build on qualitative data [4]. A CI lays out this qualitative research methodology. Contrary to usual methods, it provides methods for gathering data that a design team needs. The work models of relationship characterize the structure of the user's interactions. This research paper presents four work models which represent key aspects of user interaction and work engagement that the contextual design team needs to take into notice and consideration for in their designs when building for a work engaging environment.

\section{RELATED WORK}

Initially, a detailed study of Twitter was conducted by scrutinizing Twitter's official blog. Several design articles $[16,22,29]$ published by them were also read which helped understand the product specifications and user experience. For example, it is observed that upon landing on a profile, user's first action was to navigate through photos in order to quickly get a visual sense of the account [10]. Such imperative study helped analyze user's desires, motivations and needs before visiting selected users.

Thereafter, several research papers $[31,8,4,28,1]$ related to Twitter, Contextual Inquiry (CI), Contextual Design, interaction design, ethnographic observations and work engagement were referred for the study. The first study proposed an insightful classification of different users that were identified on Twitter based on 716 user profiles and Tweeting behavior information [31]. It classified the users 
into two broad categories i.e. Real users and Digital actors, and then further divided them into six subcategories. Thus, this categorization helped better understand user roles as well as flow of communication among these users with Twitter.

The user-centered design process of Contextual Design was referred for a strong foundational understanding of the methodology to be conducted. It presents the aspects needed to introduce the fundamentals of CI and work models [4]. This paper also demonstrates the four principles for adaption of the $\mathrm{CI}$, which have been taken into consideration.

The next study conducted a Contextual Enquiry and then built a prototype with Contextual Design of Intelligent food carrier in domestic refrigerators [19]. It determined the food item on basis of the weight and helped arrange food in the refrigerator wisely. This study also highlighted several vital aspects and requirements for refrigerator usage in Indian households.

Another study conducted two CIs of a paper-based artifact that many system developers wanted to replace with computerized variants [9]. This study took into consideration the air traffic control flight progress strip, a small paper strip used to track a flight. It notes how failure of ethnographers and developers to get along, or even understand each other, is common. It also stresses the importance and inclusion of second-order analytical work in CI.

Another insightful study in context of Indian users was that of the adoption of Automatic Teller Machine (ATM) in Mumbai, India [8]. Use of affinity diagrams for analysis revealed interesting ethnographic contradictions. There were 20 ATM users involved and the data collection identified the cultural traits associated with the power distance, collective orientation and communication boundaries. Thus, it demonstrates the role of the cultural background of the user and their expectations from the system while reflecting the resultant behavioral properties.

Major papers related to work engagement were referred from the literature $[1,5]$. According to Bakker and Leiter, "Work engagement is a positive, fulfilling, affective-motivational state of work-related well- being that can be seen as the antipode of job burnout" [1]. It is observed to serve as an application of simple tasks with a positive and affective state for work related expenditure. Additionally, when employees are engaged to their work, they find it enjoyable and reflect effective results. Considering this a few organizations support and promote the enhanced state of work engagement. Modern organizations expect their employees to be proactive by taking responsibility for their own professional development [2]. Hence, adaption of work engagement policies may offer organizations a competitive advantage.

\section{METHODOLOGY}

Eliciting essential data from users is a complex task. Merely interviewing the users is not a panacea. Neither is getting abundance of human factors involved in the early stage. Finding the right combination to get accurate work models was a challenge. The research team consists of three usability experts, who are actively involved in UI design as well as usability research. This usability research consists of four major steps as discussed further:

\subsection{Contextual Inquiry for collection of data}

A CI is usually performed in the Requirement Engineering stage and literature demonstrates that gathering good data at this stage is inherently difficult [3] and really challenging [4]. Various data gathering techniques were combined during Inquiry for this study. The technique involved preparing questionnaires, contextual interviews and naturalistic observations to explore employee use cases with Twitter in their work environment

\subsection{Development of work models}

Work models explore various types of users, their roles, primary and secondary actions, major and minor influencers of cultural traits $[4,6]$. In this research, work models address Twitter in general. Four work models have been developed which include the flow, cultural, artifact and sensory models.

\subsection{Pragmatic analysis of data}

It includes the extended analysis for higher accuracy of expert understanding of the data collected. It also includes demographic methods and programmatic means of validating data against the data collected through user's interaction and intuitive judgment. This section serves as the second-order analysis of the collected data.

\subsection{Deriving aspects from the Contextual Inquiry}

Certain interesting insights were discovered after collecting and analyzing the data. These aspects have been considered for extensions of the dimensions of work engagement Cognitive, Emotional and Physical dimension. They serve as the initial seed for future contextual designs. In the future, these aspects can be used for understanding the requirements for a regional / local design, which would also potentially be work engaging for employees and users.

\section{CONTEXTUAL INQUIRY}

Before conducting the $\mathrm{CI}$, the research team had to identify potential users who would be best fit for the study using a convenient sampling method i.e. people of acquaintance. For comprehensive understanding of Indian users, user categories on Twitter were identified as discussed ahead in the section Flow Model. A total of 18 users were considered for the study; including 5 users from each of the three categories under Real Users, and one actor from each of the three categories of Digital Actors.

The four principles of Contextual Inquiry were taken into consideration: context, partnership, interpretation, and focus $[4,14]$. The context of the users being the demographic information and the context of their work environment. All of these users were from IT companies in India. Their age group ranged from 22 to 46 years old. Majority of the users were male, at the minimum had graduated from college and had used Twitter for at least a year. Two of the users were senior managers while the others were employees. A sense of partnership was formed between the research team and the user to explore issues faced while using Twitter in their workplace together. Interpretation being the assignment of meaning to the observations [4] propelled this research to derive interesting aspects, while basing its focus of elucidating the issue of work engagement. 
A few user identities have been kept confidential. Preparing structured and relevant questionnaires was considered prior to visiting them on site, which formed the basis for the work models. Before visiting them, a few days earlier, they were informed about the procedure of CI and how the study would be conducted at their workplace/company. These users were observed using Twitter in their workplace because one cannot fully understand what people are doing or why they are doing it unless one can see and perhaps experience the interrelated conditions in which these people work $[15,26]$ or use Twitter.

\subsection{Contextual Interview questionnaire:}

The CI questionnaire mainly addresses the three dimensions essential for Personal work engagement as introduced in previous section. A series of essential questions were asked during the elicitation process of the Contextual Interview out of which a subset of these questions are discussed below. Each of the responses to these questions generated either qualitative or quantitative type of data for analysis. Most quantitative responses had the options in form of the 5 point Likert scale - Never (1), Occasionally (2), Fairly (Many Times) (3), Very Often (4) and Always (5). The initial set of questions extracted the cognitive aspect - conscious understanding and thinking of the user [12]. One such question being "How often do you find yourself mindlessly surfing and procrastinating on Twitter while indulging in meticulous work?" which subtly indicated user's mental state of commitment towards work. The next set of questions facilitated the understanding of users sentiments and feelings during work and extended the emotional aspect. Questions like, "Do you find the restriction of Twitter in your workplace or company frustrating and impacting your psychology during work?" highlighted whether there was an inclination towards conflict of interest of work. Finally, the last set of questions checked for the contribution of physical artifacts to the productivity and effectiveness of user's work - "Do you prefer smartphone over desktop for using Twitter at your workplace?"

The user interaction was not limited only to this questionnaire; but also had discussions on other aspects such as checking for preferences such as following international users over native users and triviality of Retweeting over Tweeting. It was also found that the direct messages that were sent by the users, while at their workplace, were informal in nature.

\subsection{Affinity Diagraming}

In order to understand and group information from all the visits made to the users, Affinity diagrams are used.

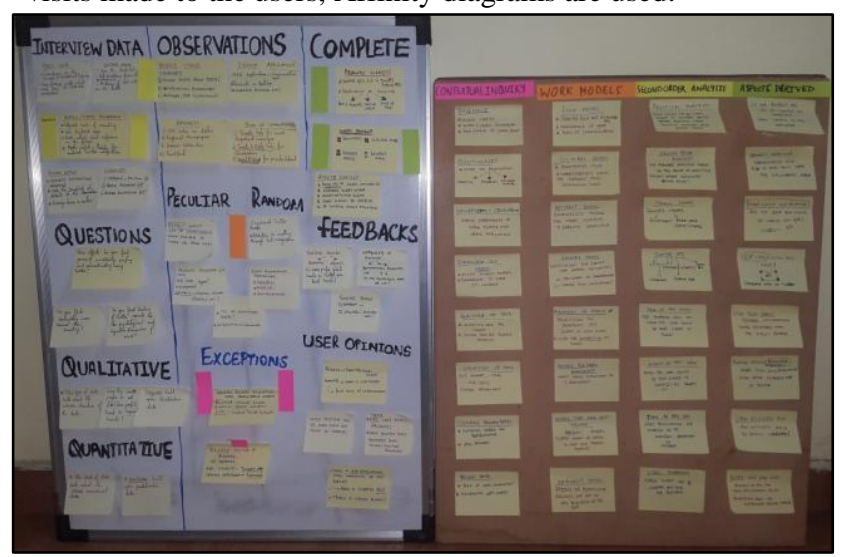

Figure 1: Forming the Affinity diagram
This methodology [4] involves the use of sticky notes and serves as the paragon for effective categorization after the brainstorming session. As shown in the figure 1, lists of items were written down on sticky notes, for example, related functions or features of Twitter that were essential to the study and then were organized by grouping them. They uncovered some interesting scenarios such as use of smart watches for Personal and Professional user differed drastically. The former used it more while driving to and from their workplaces and, the later used it more in their homes.

\section{WORK MODELS}

In order to study and document the way people use Twitter, certain work models have been inherited. These models help build guidelines for contextual design and guidance for effective work engagement in different environments. They represent the key aspects of work engagement that, design teams need to account for in their design. Synergy of users with Twitter to communicate ideas, opinions and views are also reflected in these models. Four work models, namely, Flow model, Cultural model, Artifact model and Sensory model have been developed during CI. Developing these models requires rigorous onsite observations, and obtaining vital insights out of data analysis. It is a time consuming process. The physical model captures the places where work occurs [15] and is used when the product is physically available in that place. In this case, artifacts compensate virtually for Twitter's physical existence and hence, the physical model was not considered in this research. A Sequence model considers a trigger or uniquely states the intent of the sequence, but this doesn't work for Twitter since most activity can be carried out without a fixed sequence. The other four models are discussed ahead.

\subsection{Flow Model}

Flow models target on diagnosing responsibility and coordination of the types of users. It identifies the flow of communication and user interaction with Twitter $[4,6]$.

\subsubsection{User Types}

Based on the user profiles and their Tweeting behavior, Twitter users are categorized into six different types [31], belonging to two major parent categorizes, Real users and Digital actors. These users are described below:

\subsubsection{Real Users}

These are the human users. They register with Twitter by creating an account having a unique Twitter id. The next-level three types of Real users are discussed ahead.

Personal users - These users are real people who used Twitter for Personal use and don't have specific intentions to Tweet about any particular subject. Active Personal users tend to Tweet about current happenings in their lives, trending stories, latest news that create sensation among their friends or related user group. Professional users - Professional users are the real users who Tweet with professional intent on Twitter. They share useful information and involve in healthy discussions about their area of interest and expertise. These are highly interactive and follow many users concerned with their profession. Business users - Business users differ from Personal and Professional users as they follow a marketing and business agenda on Twitter. Their profile and description clearly depicts their intention and motive. The key factors that determine Business users are frequent Tweets and lesser interaction. 


\subsubsection{Digital Actors}

These users are Twitter bots created by Real users that are made to function programmatically through official Application Program Interfaces (API) provided by Twitter. Sometimes, they tend to backfire as with the case of Microsoft's Artificial Intelligence Twitter bot, Tay. Within 24 hrs of its activity, it started Tweeting awful and racist Tweets [24].

Spam users - These are Twitter bots with intentions to Tweet or reply unrelated content to Real users. Twitter listed spam accounts as a "risk factor" in a recent finding, which estimated that 5 percent of active accounts are fake [13]. Feeds/News - These bots broadcast news with their Tweets. They usually tend to be bots created by news organizations. Most Tweets contain Universal Resource Locators (URL), images and Graphics Interchange Format (GIF) of the recent happenings. Viral/Marketing services - These bots are programmed to be intelligent and used as services to spread the words amongst users about the brand they are endorsing. Bots, which claim to automatically follow back when followed, were noticed. A few bots are monetized by selling followers.

\subsubsection{Derived Flow Model}

In figure 2, the two actors, Real users and Digital actors depicted on each side, are the major parent categories of Twitter users. The frequently performed actions on Twitter are enclosed in ellipse and collectively their activities are enclosed in the rectangle. The arrowed links depict the probable actions each user could perform since all the users are liable of performing all the actions stated inside the rectangular box. The methods for performing the tasks are inside the ellipse. Multiple parameters in the ellipse have been considered for each of the actions, which help evaluate the user's pattern and behavior.

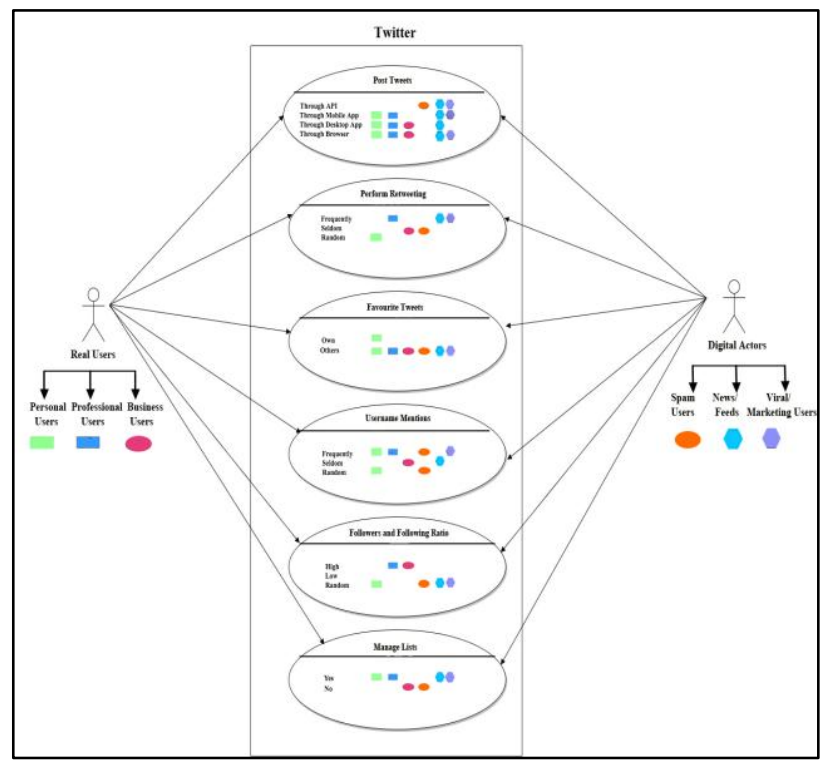

Figure 2: Flow model for Twitter users

In few cases, parameters besides the method of performing the action, have been taken into consideration like, binary parameter i.e. yes or no decisions. The Personal users and Professional users are represented using green and blue rectangles respectively. The Business users and Spam users are depicted with deep pink and orange ellipses respectively. Finally, the digital users categorized as Feeds/News and Viral/Marketing services are shown by a blue and purple colored hexagons. The following section provides summary of the behavioral assets found with three users of each type i.e. total of 18 users.

\subsubsection{Workflow}

Workflow of the flow model emphasis on the state of people's work, their interaction and coordination ensuring the task is completed.

\subsubsection{Favourite/Like count}

Based on observation and through sentiment analysis APIs, it was found that most Personal users often liked their own and others positive Tweets. Some of them thought that more the number of likes their Tweet gets better would be the perceived quality of the Tweet, which isn't necessarily always true. All the other users do not prefer to like their own Tweets, especially Business users.

\subsubsection{Username mention frequency}

Personal users, Professional users and marketing bots publically@mentioned other Twitter users frequently and randomly while Business users and Feeds/News bots rarely engaged publically into conversation by seldom mentioning anyone. After analyzing their Tweets, a lot of them hadn't replied back to users who commented/replied on their public Tweets. The exact opposite was seen in case of Personal users profiles, who were more willing to express themselves and took time out to respond.

\subsubsection{Followers and Following Ratio}

Professional users and Business users followed very less number of people, but more number of people followed them. Personal users, Spam users, Feeds/News and marketing bots had random ratios observed for their count for followers and followees.

\subsection{Cultural Model}

The cultural model identifies the cultural traits of Indian Twitter users. People's work productivity is highly influenced by the culture they follow and the culture that the people around them follow. It defines expectations, desires, policies, values, and the whole approach people take to their work [4]. This model describes the aspects in which Indian users differ from other international users. The scope of the notion of culture in this paper is restricted to the traditional culture of India and work culture of the workplace. Individuals are the several types of Twitter users, which have been categorized previously in the Flow model. The Influencers that influence a certain culture may provoke changes in Individual's actions. The findings of the primary ethnographic study are reflected further:

\subsubsection{Differences in work culture}

A previous study with 572 Dutch employees showed that burned-out and engaged employees can be distinguished from their counterparts on the basis of their personality and temperament [32]. One of the studies [18] found that "Most of the Indians think workplace as an opportunity to build their future and put forth extensive efforts to climb the corporate ladder and earn monetary benefits. This also makes them encounter a lot of pressure, as the demands at work place and family are almost opposite". This study found that to counter this work pressure, sometimes they end up Tweeting their agony on Twitter. Retweet like "i love that $i$ get hit on so often at work i have code words for my managers to come up and get rid of them :-)" and public Tweet like "Only been at 
work for an hour, And I'm already tired asf!" from the employee reflected their temperament towards work.

The study [18] also found that "Western users take lunch break for half an hour. On the contrary, in India, people do not adhere to the timings. No doubt, they work for long hours approximately for 10-12 hrs a day and sometimes even on weekends, but take long tea breaks and lunch breaks" [18]. In this CI, users were found spending time discussing events like trending topics on Twitter as well as news related topics during these long lunch breaks leading to delay in getting back to work.

\subsubsection{Minor Influencers}

Tone of user's Tweet and organizational workplace policies play the role of minor influencer. These are subjective and may change from time to time. Some workplaces have restriction on the use of Twitter and other social networking sites as part of their strict workplace policies. Contrary to most, a few organizations had strict policies of not allowing Smartphones in their work place and hence, these users were left with the only option of using Twitter in their web browser. These elements may have influence on an individual's work culture.

\subsubsection{Major Influencers}

\subsubsection{The Time Zone Dilemma}

Some users found it confusing and didn't understand the changing behavior of Tweet's information or metadata. They thought that once they log in and set their preferred time zone for the account, Twitter would remember their preferences and would show the same time zone for the Tweets upon logging out of the account. Contrary to that, Twitter defaults the Time Zone to (GMT-07:00) Pacific Time (US \& Canada).

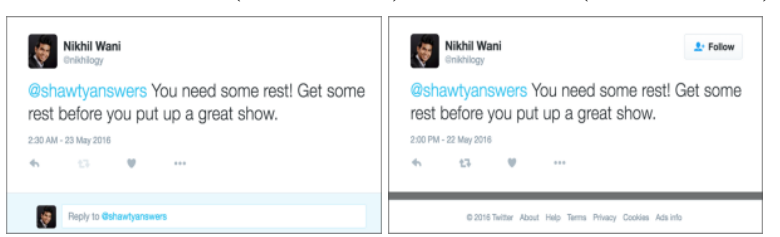

Figure 3: Logged In on the left, logged out on the right.

Hence, when an user logs into Twitter with Time zone settings configured to (GMT+05:30) New Delhi and tries to view the Tweet's information while still being logged in, he/she would be presented with the time and date in context of IST (Indian Standard Time), which is right, but when he/she logs out and checks the same Tweet's information, they are presented with a different time and date than the original. This different time zone is the (GMT-07:00) Pacific Time (US \& Canada). For example, Tweet shown above was made on $23^{\text {rd }}$ May, 2016 at 2:30 AM, once logged out, the same Tweet shows $22^{\text {nd }}$ May, 2016 at 2:00 PM. Hence, the local scope changed when it was presented globally and violates the integrity of the user's Tweet. This is a genuine usability issues which can be approached by specifying the Time zone along with the time in the meta data. This would eliminate the slightest chance of confusion and would provide a smooth design with integrity of data.

\subsubsection{Use of alternative notation for "at replies".}

Tweets that start with an '@' and then the username are also called as "at replies"[16]. Twitter organizes Tweets in three tabs namely, "Tweets", "Tweets and Replies" and "Media", which a few Personal users found it initially confusing. When a user's profile is visited, by default he/she sees the Tweets tab, which presents the user's timeline of Tweets and is without replies that is, the Tweets that begin with an (a) username won't be shown. This makes it easier to understand the kind of content the user wants to share and what one sees if one follows them. Replies which have @ yourusername in the Tweet and are from people one is not following, are placed in the "Tweets and Replies" Tab. If the users follow the person who @ replies them, then that Tweet would appear in that user's main timeline, but he/she will always see them in the "Tweets and Replies" tab, whether he/she follow them or not and unless he/she has blocked them.

Today, @ replies are a critical part of how Twitter works [16]. Hence, it was found that in order to avoid the Tweet from being displayed in the secondary "Tweets \& Reply" tab, user's started the Tweet with a character and then @mention. This would force the Tweet to be displayed in the "Tweets" tab only. This tab being the default and primary landing tab, user's prefixed a "." character at the beginning of the Tweets, which was the content they wanted to share with their followers. These prefixed Tweets would be seen by their followers in their main timeline, which otherwise wouldn't have been shown if the Tweet landed in the "Tweets \& Replies" tab.

\subsubsection{The Devanagari script syndrome}

India's diversity is reflected by its multi-linguistic culture. Through legislation, each of the 29 states in India have the liberty and power to specify their own official language(s). Hence, there are 22 officially recognized languages in India. Nine states and two union territories have Hindi as their official language making it the most commonly used language followed by English and other 16 other regional languages. Devanagari script was found to be the most commonly used script amongst Indian Twitter users. Several Indian languages, including Hindi and Marathi use this script. At times, users preferred to use it over the Roman script which is used to write English. In regional context, users prefer using Devanagari, but in global context they preferred English as their default language on Twitter. These details can be taken into consideration in the future, when text-mining applications are built to analyze the behavior of the Tweet in regional scripts.

\subsubsection{Fan accounts with their Bollywood and Cricket affection}

During this study a subcategory of personal users was also found. These users called themselves fans of celebrities. Fan accounts exist in all countries, but in Indian context, plentiful of accounts were found, which specifically pondered, to Bollywood celebrities and Cricketers. Following is the division of this subcategory:

\section{a. Fan accounts with username as Bollywood celebrity.}

These account were real users, mostly Personal users who renamed their online identity by tweaking their usernames to celebrity name/nicknames.

\section{b. Fan accounts with Display Picture of Bollywood celebrity.}

These users had celebrities' picture as their own account's display picture. Their Tweets talk only about the celebrity 
they have as their display picture. Accounts like these mostly promote the mentioned celebrity and their ventures.

\section{c. Fan accounts turned into fan clubs/fan pages of Cricketers}

These types of user accounts are created by real users with a single intention of finding and attracting other fan accounts to collectively Tweet, favorite/like and Retweet the celebrity accounts. They also lure other accounts by claiming to follow back the users that follow them.

\subsubsection{Preferences of Global trend over Regional trend}

Trends, which are presented in the form of lists, help users discover the emerging topics people are talking about on Twitter [29]. In order to show the trends that matter to the users, Twitter's algorithm tailors them based on the current location of the user and the people they follow. It allows the user to select one of more than 150 locations available. The default is the user's regional location. It was observed that users preferred to see a general list of worldwide trends than regional trends. The users used the option "Get the tailored trend" button, which sets the trend to worldwide trends. Occasionally, when they wanted to see what was happening around a particular city, they had the option of filtering trends.

\subsection{Artifact Model}

This is used to understand the existence and use of artifacts that are used along with Twitter. It captures the things people use or create when doing work [15] in this case, when using Twitter in their workplace. Hence, they have the potential to affect users' effective and efficient working environment. Important Artifacts Identified were - Regional Offline calendars and Digital Calendars, Offline Diary notes, Smartphones and news feed Applications, Regional offline newspapers, QR codes, Twitter tools such as Tweetdeck and IFTTT for power users of Twitter. India has plethora of regional festivals during which users tend to post wishes on Twitter. Acknowledging this fact, the existence of regional offline calendars was found on user's workplace desk boards. These calendars had markings of regional festivals.

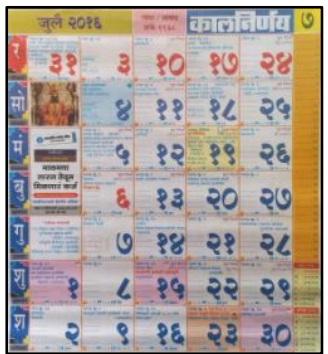

Figure 4: Regional offline calendar [20]

They possibly served to be of great importance to the marketing team of the organization, which posted Tweets during these festivals. Users also referred and relied on online calendar applications such as Google calendar to schedule reminders for Tweeting important Tweets that had planed to Tweet later at some specified time.

Twitter also serves as a large platform for personal micro blogs, for which users tend to prepare a personal diary to note interesting things they observed throughout the day when they were offline and refer the same while Tweeting. Contrary to most, a few organizations/companies had strict policy of not allowing Smartphones in their workplaces and hence, these users were left with the only option of using Twitter in their web browsers. When users used smartphones for Tweeting, they often had technology related Feeds/News application such as Hike news and Flipboard installed. Users were very likely to share these Feeds/News notifications as Tweets. Offline regional newspapers and English newspapers were observed on their desks, which served as a stimulus for Tweets pondering to what was happening around them.
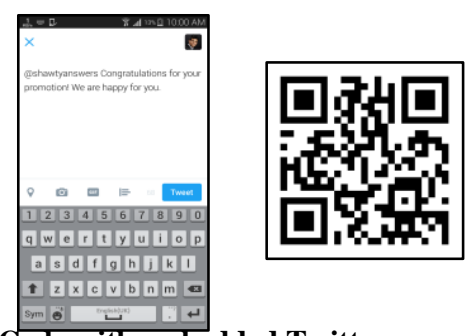

Figure 5: QR Code with embedded Twitter message (left), Twitter app screen upon scanning the $Q R$ code (right)

QR codes and Near-field communication (NFC) stickers with Twitter data embedded were observed on tables. Upon scanning the QR codes it would redirect the user to the Twitter's mobile application with the embedded message. Automated Tweets, which needed to be posted every week, were embedded in the QR codes. Some users found Twitter's interface complex and confusing and hence, switched to TweetDeck, a powerful Twitter tool for real-time tracking, organizing, and engagement. TweetDeck is now a subsidiary of Twitter. Automation apps such as IFTTT were used by user's to create recipes in the app, which automated the posting of their Tweet on a custom event and saved their time.

\subsection{Sensory Model}

This model includes materialistic components that advance the experience of the users [19]. In case of Twitter, sensors alert the user when an event occurs. These censors can be a form of light, blinking LEDs specifically in case of Smartphones. They can also be set to a form of sensation such as custom vibrating pattern on a device. Events can arise as a result of direct messages, @ mentions from users one follows, Retweets, likes, polls, new followers, crisis \& emergency alerts and other types of actions one can elect to receive [23]

\subsubsection{Mobile Notifications}

Smartphone notifications of Twitter can be received in two ways - with and without the official Twitter mobile application. Users can opt for one of these two ways, which are detailed as follows:

\subsubsection{SMS Text Notification}

Notifications can be delivered as SMS text messages to users who attach phone numbers to their account. This is the direct method of receiving Twitter event notifications. Short codes provide two-way communication i.e. posting a Tweet and receiving Tweets if possible. Long codes provide one-way communication i.e. only sending of Tweets is possible. Additionally, international messaging rates may apply when using these long codes. Users rarely used these codes and if at all when they did use, they preferred the short code method over the long code.

\subsubsection{Push Notifications}

When a user isn't actively using the Twitter app, these notifications allow notifying the user of the new message. 
They appear in the status bar of the device. Most users were found using the default vibration notifications on Android and the single beeps on Windows devices.

\subsubsection{Smart watches}

Most Wearable smart watches offer notifications and voice commands to its users. Moreover, Twitter's research team found that early adopters on Twitter are $75 \%$ more likely to have recently purchased a smart watch [22]. During this study, it was found that about three fourth of the convenient sample, especially the tech professionals, used Pebble ${ }^{\text {TM }}$ smart watches or Xiaomi Mi band in their workplace. While it was obvious that they didn't want to be notified actively the entire time during their work, but interestingly they used these watches in their cars while driving to or from their workplace and didn't want to miss out on important alerts on Twitter while they were away from their phone.

\section{PRAGMATIC ANALYSIS OF DATA}

A practical approach was used in order to study the demographic information of the convenient sampled users for an effective CI. This serves as the second-order analysis of the data. Python scripts were written using the Tweepy library, which fetched the users' public Tweets. Thereafter, scripts were written which computed their frequency for the past eight months. Analyzing demographic data by programmatic verification and validation leaves no scope of error, rather than user's second-guessing based on their intuitive memory of their Tweeting behaviors. Tweets made by users starting from 31st October 2015 to 22nd May 2016 were considered. It was decided to observe the Tweeting pattern by classifying them on the basis of, Weekly frequency of Tweets, Monthly frequency of Tweets and Tweets during the day. This data was publically available and hence open for analysis.

\subsection{Demographic Business Intelligence}

\subsubsection{Weekly frequency of Tweets}

Weekly statistics were drawn out from the user's account. The graph below in figure 6 shows the comparison of frequency of Tweets posted by users in 32 weeks. Each day of the week is taken into consideration for finding insightful information out of the data obtained. In order to protect user identity, alias for the original names have been created in this section - @ma and @ng were identified as Professional users and @Iam was identified as a Business user.

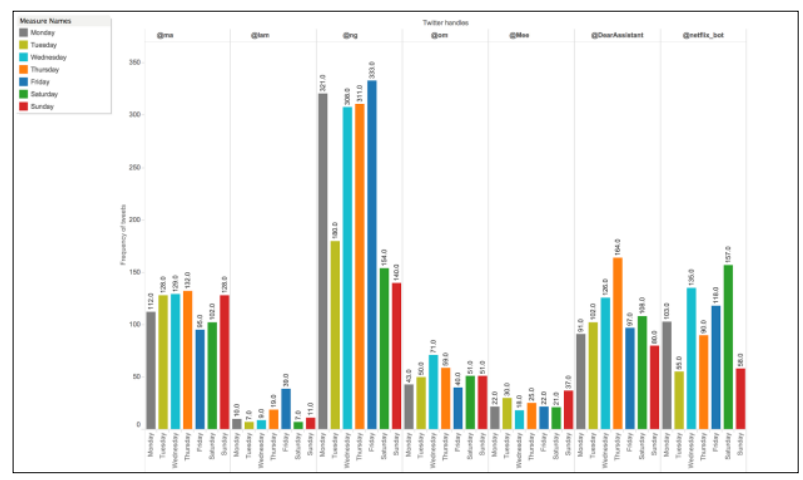

Figure 6: Users Tweeting frequency for the past 32 weeks

Most Indian Professional and Business users were likely to Tweet either on Thursdays or Fridays as the graph suggests. They seemed to post Tweets often on the weekends. @DearAssistant and @ netflix_bot were the identified bots which were more active on Wednesdays comparatively with an average 130 Tweets. Personal users like @om and @Mee were found to be active on Tuesdays and Thursdays. Professional users tend to Tweet a lot more than any other users. As shown in the graph above, @ng had a maximum of 333 Tweets in 8 months on Friday, which was the highest amongst all the other users that were observed.

\subsubsection{Monthly frequency of Tweets}

As shown in figure 7, Twitter bots Tweeted the most in November and December. This behavior can be justifiable because most local ecommerce websites in India, like Flipkart and Snapdeal offer lucrative discounts on products during their sale period, which starts in October every year [21].

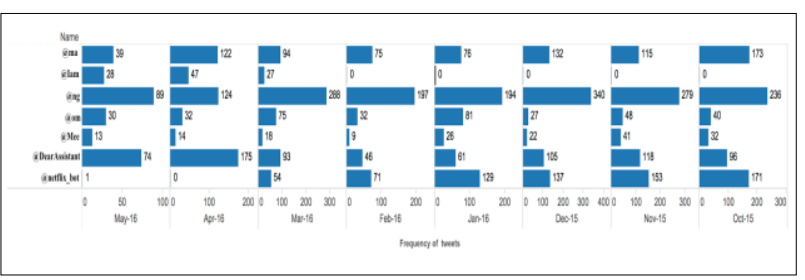

Figure 7: Users Tweeting frequency in the past 8 months A few Business users who had just begun using Twitter were also taken into consideration. Statistics of @Iam reflect their steady start on the Twitter platform. Most of their Tweets were either Retweets or Tweets related to their organization. In this case, it was found that the user were Tweeting and Retweeting about affiliation products of Microsoft, as he was an employee of the organization.

\subsubsection{Tweets during the day}

Interestingly, five out of seven Indian users, as shown in table 1, had the most Tweets posted in the Afternoon, which at most corporates are occupied by lunch and breaks.

Table 1: Users Tweeting frequency throughout the day

\begin{tabular}{|c|c|c|c|c|c|c|c|}
\hline & \multicolumn{7}{|c|}{ Frequency of Tweets } \\
\hline Name & M/D & EM & MM & $\mathbf{A F}$ & $\mathbf{E V}$ & NG & $\Sigma$ \\
\hline @ma & 75 & 122 & 123 & 134 & 212 & 160 & 826 \\
\hline @Iam & 13 & 7 & 10 & 26 & 227 & 7 & 85 \\
\hline$@$ @ng & 6 & 174 & 576 & 677 & 254 & 60 & 1747 \\
\hline @Mee & 21 & 12 & 71 & 96 & 77 & 88 & 365 \\
\hline @om & 29 & 8 & 20 & 58 & 16 & 44 & 175 \\
\hline $\begin{array}{l}@ \text { Dear } \\
\text { Assist } \\
\text { ant }\end{array}$ & 178 & 141 & 55 & 147 & 142 & 106 & 769 \\
\hline $\begin{array}{l}\text { @ netfl } \\
\text { ix_bot }\end{array}$ & 1 & 0 & 0 & 0 & 715 & 0 & 716 \\
\hline
\end{tabular}

Index: M/D = Morning/Dawn (00:00 to 4:59), EM = Early Morning (5:00 to 8:59), MM = Mid - Morning (9:00 to 11:59), $\mathbf{A F}=$ Afternoon (12:00 to 16:59), $\mathbf{E V}=$ Evening $(17: 00$ to $20: 59), N G=$ Night $(21: 00$ to $23: 59), \Sigma=$ Total in 8 months.

Hence, most users find themselves free to post Tweets. Statistics of bots revealed that their nature of Tweeting on any particular day was unpredictable. Some days they wouldn't post a single Tweet and plummet, while on the other days they seem to Tweet throughout. As reflected by Personal users statistics in the table above, these Indian users are most likely to post Tweets in the Afternoon i.e. after 12:00. Out of the convenient sampling of 18 users, 13 users were observed with the same results. 


\subsection{Random Sampling analysis}

A random sample of 2,000 Twitter profiles of Indian employees who work at various IT and non-IT companies were examined. This resulted in understanding reasons for employee's venting out satisfactory or dissatisfactory opinions about their work, in general, through their Tweets. Out of a total of 72,000 Tweets processed, more than 4,250 Tweets had "work" related and occurring phrases. The top keywords found amongst these phrases were "work", "workplace", "work engagement", "job satisfaction", "burnout" and also consisted of other minor phrases. Phrases that ended with cause relationship words were also considered, such as "therefore", "thus", "hence", "consequently", "as a result". Phrases with sentiments were given additional consideration and were investigated to derive factors that influence the engagement of work. For example, "I hate my workplace because" and "I love my workplace so" contain both positive and negative sentiments.

\subsection{Factors that influence work \\ engagement}

Factors include the points derived from the Tweets. These factors have been obtained after processing the Tweets, which had 'work' related and occurring phrases as stated earlier. The following major points were included in dissatisfactory Tweets that indicate negative sentiments: disliking their colleagues, restriction on Internet usage during work hours, lack of Infrastructure for entertainment breaks. The following major points were included in satisfactory Tweets that indicate positive sentiments: Burnouts due to excessive work demands lead to users taking mental break on Twitter. This ameliorated the possibility of negative health related outcomes arising from burnouts, Searching with \#PuneTraffic, or any city traffic during work hours on Twitter would provide the employee with real time traffic updates from authentic Twitter accounts. This may help the employee concentrate on their current work and alleviate future worries.

\section{ASPECTS DERIVED FROM CONTEXTUAL INQUIRY}

In this section, holistic inference and extraction of certain key points has been performed which support the CI and work models. The Contextual Data produced from a CI can be used to write and buttress the generation of user personas [15]. Hence, the aspects derived from this research can also be used to write user personas of Indian Twitter users. These important aspects have been summarized as follows:

- Use of artifacts like QR codes and NFC stickers with pre-defined quotidian messages to be posted were found on user's worktable, which interestingly provided an alternate method of Tweeting. This improved effectiveness by saving the time needed to type for circulating a broad message that was to be passed around in the workplace.

- About $\mathbf{7 2 \%}$ Indian Twitter users, specifically Personal users, are most likely to post Tweet in the Afternoon i.e. just before taking the lunch break around 1 PM (IST), implicitly suggesting that they opt for a mental break favorably on Twitter during work hours. "I am always occupied with some or the other task throughout the day because of multiple project deadlines...feels like I need to get up from my desk... and breath some fresh air into my life everyday! I find that exit window while reading witty and funny banter on Twitter". This quote from one of the senior employee from a reputed IT firm, while conducting the onsite visit, subtly indicated that excessive work demands sometimes lead to burnouts which is inevitable and hence to ameliorate negative mental health related outcomes like depression, users take mental break on Twitter during work hours.

- Indian Twitter users posted lesser Tweets on weekends than on weekdays with a ratio of $1: 3$, indicating their strong inclination towards spending quality time away from work and preferentially clocked lesser activity on Twitter.

- Disliking their workplace environment, which includes cornering colleagues, counter intuitive infrastructure and strict quixotic policies rather than usage of Twitter during work hours was found to be the cause of deterioration in positive and fulfilling state of their work. "We are not permitted to access our smartphones inside the office and even if we get it in our cameras are white taped to prevent any recordings, let alone using Twitter on smartphones! Desktop proxy and chill!" exclaimed one of the users when asked about his Twitter usage, who was a fresher working for an IT consultancy company. This aspect also resonated with the findings published by Proskauer, in their third survey stating that in 2014, 36\% of employers actively blocked access to social media sites, compared to $29 \%$ in 2012 [28]. According to above derived aspect, the trend of blocking Twitter in Indian workplaces has still not been abolished on a large scale.

- Twitter's claims that their users were $75 \%$ more likely to have recently purchased a smart watch than users of other social networks [22]. Considering this, it was found that even Indian employees or Professional users who were early adopters of Twitter had recently purchased a smart watch because they found the interface simple, intuitive and faster to use. The inherent human ability to process lesser information faster, in this case displaying a single tweet from their feed at a time, on a standard smart watch screen resolution of $320 \times 320$ pixels worked well with almost all the users in the context of their usage during work than using it on a smartphone.

- Indian users cultural peculiar traits included the prioritization and special inclination towards regional languages and Devnagari script for Tweeting during festivals and special occasions rather than the established Roman English scripted Tweets even when they had the option of Tweeting the same Tweet in the defaulted Roman English script.

- Increased interest in Tweets related to Bollywood and Cricket formulated a novel line of thought for the users' psychology. Out of all the topics and sports events, these were the fastest emerging ones. This aspect compliments a recent study that stated Indian Premier League was found to be the fastest growing sports league on Twitter [11]. It was observed that during Cricket matches, some users frequently checked Twitter feeds after short intervals for live scores and updates while they were on their work desk. 


\section{CONCLUSION AND FUTURE WORK}

The comprehensive Contextual Inquiry and four related work models have highlighted numerous important aspects and communicated vital preferences of Indian Twitter users leading to an opportunity to help elucidate the issue of work engagement. These aspects have been derived keeping in mind the three dimensions of work engagement - Cognitive, Emotional and Physical engagement. A second-order analysis of the data was computed by programmatic means for at most accuracy of the valuable derived aspects. These Twitter work models enlightened certain esoteric ways of Twitter usage in IT and non-IT companies, while derived aspects reflected the inferences deduced from those models. This research work can moreover be an imperative basis for writing user personas of Indian Twitter users.

In future, this study can be extended in the making of work engaging Artificial Intelligence Twitter bots. These bots should base their Contextual Design on CI, related work models and derived aspects for Ethnographic alignment of its design. This Ethnographic study can be extended for Contextual Designs of regional microblogging sites as well. It will help Twitter users engage with their work better and use it effectively. Shawty, an interactive Q\&A Twitter bot developed by our team builds upon the aspects that have been derived in this paper. The initial beta version of the bot has been deployed as@shatyanswers.

\section{REFERENCES}

[1] Bakker, A. and Leiter, M. Work engagement. Psychology Press, Hove [England], 2010, 1-2.

[2] Bakker, A. and Schaufeli, W. Positive organizational behavior: engaged employees in flourishing organizations. Journal of Organizational Behavior 29, 2 (2008), 147-154.

[3] Bashar Nuseibeh and Steve Easterbrook. 2000. Requirements engineering: a roadmap. In Proceedings of the Conference on The Future of Software Engineering (ICSE '00). ACM, New York, NY, USA, 3546. DOI=http://dx.doi.org/10.1145/336512.33652

[4] Beyer, H., Holtzblatt, 1998. Contextual Design. Morgan Kaufmann, San Francisco, CA. 46-120

[5] Bakker, Arnold B., and Evangelia Demerouti. "Towards A Model Of Work Engagement". Career Development International 13.3 (2008): 209-223. Web.

[6] Chinnapattan P. Contextual inquiry research: A guide. Webcredible.com, September 1, 2011. http://www.webcredible.com/blog/guide-conductingcontextual-inquiry-user-research/

[7] Company|About.about.twitter.com,2016. https://about.twitter.com

[8] De Angeli, A., Athavankar, U., Joshi, A., Coventry, L. and Johnson, G. Introducing ATMs in India: a contextual inquiry. Interacting with Computers 16, 1 (2004), 29-44.

[9] Dekker, S., Nyce, J. and Hoffman, R. From contextual inquiry to designable futures: what do we need to get there?. IEEE Intell. Syst. 18, 2 (2003), 74-77

[10] Designing the new Twitter profiles | Twitter Blogs, Rao, S. Blog.twitter.com,2015. https://blog.twitter.com/2015/designing-the-new-twitterprofiles

[11] Devgan, A. IPL is the fastest growing sports league on Twitter. International Business Times UK, May, 16, 2016. http://www.ibtimes.co.uk/ipl-fastest-growing-sportsleague-twitter-1560256

[12] Helander, M. Handbook of human-computer interaction. North-Holland, Amsterdam, 1991. 3-34

[13] Here Come the Marketing Bots. MIT Technology Review, 2016. https://www.technologyreview.com/s/601225/herecome-the-marketing-bots/

[14] Holtzblatt, K. and Jones, S. (1993) 'Contextual Inquiry: a participatory technique for systems design', in Participatory Design: principles and practice, Schuler, D. and Namioka, A. (eds), Lawrence Erlbaum, New Jersey, 177-192.

[15] Holtzblatt, K., Wendell, J. and Wood, S. Rapid contextual design. Elsevier/Morgan Kaufmann, San Francisco, 2005. 123-190

[16] How @ replies work on Twitter (and how they might) Twitter Blogs. Blog.twitter.com, 2008 https://blog.twitter.com/2008/how-replies-work-on-twitterand-how-they-might

[17] Indians spend more time on Facebook, Twitter than on email: Survey - Times of India. The Times of India, 2016/ http://www.gadgetsnow.com/social/Indians-spend-moretime-on-Facebook-Twitter-than-on-emailSurvey/articleshow/12451204.cms

[18] Jhunjhunwala S, (2012). Review of Indian work culture and challenges faced by Indians in the era of globalisation. Interscience Management Review (IMR), 2(2), 70-73

[19] Kale, P., Bhutkar, G., Pawar, V. and Jathar, N. Contextual design of intelligent food carrier in refrigerator: An Indian perspective. In Human Work Interaction Design. Work Analysis and Interaction Design Methods for Pervasive and Smart Workplaces. Springer International Publishing, 212-225.

[20] Kalnirnay $2016 \quad$ kalnirnay2016.in http://kalnirnay2016.in/wp-content/uploads/2015/10/July2016-Marathi-Kalnirnay-Calendar.pdf

[21] Kumar, P., Vashisht, A., Sharma, A. An empirical study of customer confidence after big billion day sale 2014.South Asian Journal of Marketing \& Management Research 5, 4 (2015), 97-105.

[22] New research: 8 ways early tech adopters use Twitter Twitter Blogs. Blog.twitter.com, 2016. https://blog.twitter.com/2016/new-research-8-ways-earlytech-adopters-use-twitter

[23] Notifications on mobile devices. Twitter Help Center, 2016. https://support.twitter.com/articles/20169887

[24] Price, R. Microsoft is deleting its AI chatbot's incredibly racist tweets. Business Insider, 2016 http://www.businessinsider.com/microsoft-deletes-racist- 
genocidal-tweets-from-ai-chatbot-tay-2016-

3 ? $\mathrm{r}=\mathrm{UK} \& \mathrm{IR}=\mathrm{T}$

[25] Raghu, G. Flipkart's Strategy. Ssrn.com, 2015. http://ssrn.com/abstract=2546713

[26] Raven, M. and Flanders, A. Using contextual inquiry to learn about your audiences. ACM SIGDOC Asterisk J. Comput. Doc. 20, 1 (1996), 1-13.

[27] Smith, C. 170+ Amazing Twitter Statistics. DMR, Updated 2017. http://expandedramblings.com/index.php/march-2013-bythe-numbers-a-few-amazing-twitter-stats/

[28] Social Media In The Workplace Around The World 3.0. 1st ed. Proskauer, 2014. Web. 21 Mar. 2017.
[29] Tailored Trends bring you closer | Twitter Blogs. Blog.twitter.com, June 12, 2012. https://blog.twitter.com/2012/tailored-trends-bring-youcloser

[30] Ojeda-Zapata, J. Internet becomes lifeline, record. Twin Cities, Updated November, 14, 2015 http://www.twincities.com/2007/08/01/internet-becomeslifeline-record/

[31] Uddin, M. M., Imran, M., \&Sajjad, H. (2014). Understanding types of users on Twitter. arXiv preprint arXiv:1406.1335.

[32] Langelaan, Saar et al. "Burnout And Work Engagement: Do Individual Differences Make A Difference?". Personality and Individual Differences 40.3 (2006): 521532. 